\title{
Kinetic Inductance Detectors as light detectors for neutrino and Dark Matter searches
}

\author{
Angelo CRUCIANI, Elia Stefano BATTISTELLI, Ivan COLANTONI, Alessandro \\ COPPOLECCHIA, Antonio D'ADDABBO, Paolo DE BERNARDIS, Silvia MASI \\ Università La Sapienza, Rome, Italy \\ E-mail: angelo.crucianieromal.infn.it
}

\section{Fabio BELLINI, Laura CARDANI, Carlo COSMELLI, Marco VIGNATI \\ Università La Sapienza and INFN, Rome, Italy}

\section{Carlo BUCCI}

Laboratori Nazionali del Gran Sasso (INFN), L'Aquila, Italy

\section{Martino CALVO}

Institut Néel (CNRS), Grenoble, France

\section{Gabriella CASTELLANO}

Istituto di Fotonica e Nanotecnologie (CNR), Rome, Italy

\section{Sergio DI DOMIZIO}

Università di Genova and INFN, Genova, Italy

\section{Davide PINCI, Claudia TOMEI}

INFN Sezione di Roma, Rome, Italy

Large-mass arrays of bolometers proved to be good detectors for neutrinoless double beta decay (0vDBD) and Dark Matter searches. CUORE and LUCIFER are bolometric 0vDBD experiments that will start to take data in 2015 at Laboratori Nazionali del Gran Sasso in Italy. The sensitivity of CUORE to $0 v \mathrm{DBD}$ could be increased by removing the dominant source of background, due to $\alpha$ particles, exploiting the small amount of Cherenkov light emitted by the $\beta$ (signal) and not by $\alpha$ s. LUCIFER could be extended to search also for Dark Matter interactions, provided that an efficient rejection of $\beta / \gamma$ background in the energy region of interest (below $10 \mathrm{keV}$ ) can be obtained using the simultaneous detection of heat and scintillation signals. The interest in sensitive cryogenic light detectors gave birth to the CALDER project, that aims at the development of detectors with an active area of $5 \times 5 \mathrm{~cm}^{2}$ (the face of typical bolometric crystals), operating in a wide temperature range around $10 \mathrm{mK}$, and with an energy resolution at the baseline below $20 \mathrm{eV}$ RMS. We have chosen to develop phonon-mediated detectors with Kinetic Inductance Detector (KID) sensors. We have tested the first prototypes, that were able to trigger the first pulses due to cosmic rays.

Technology and Instrumentation in Particle Physics 2014

2-6 June, 2014

Amsterdam, the Netherlands 


\section{Introduction}

In the near future, bolometric experiments searching for rare events could play a key role in our understanding of neutrino physics and Dark Matter nature. The experimental sensitivity, that is mainly limited by the background due to natural radioactivity, could be enhanced by the capability to identify the nature of the interacting particle.

Bolometers are large crystals coupled with temperature sensors and operated as calorimeters at about $10 \mathrm{mK}$. They are particularly suitable for $0 v \mathrm{DBD}$ searches because they can reach high efficiency (larger than 75\%), excellent energy resolution ( $0.1 \%$ from a few $\mathrm{keV}$ to a few $\mathrm{MeV}$ ) and low intrinsic background. The lack of an active background rejection tool can be compensated using a light detector, that identifies the nature of the interacting particle exploiting the different light emission of different nature.

Two good examples to illustrate the potentiality of cryogenic light detection are CUORE [1] and LUCIFER [2], that will start taking data in 2015 at the Laboratori Nazionali del Gran Sasso (LNGS), Italy.

CUORE searches for the ${ }^{130} \mathrm{Te} 0 v \mathrm{vBD}$ using an array of $988 \mathrm{TeO}_{2}$ cubic crystals $\left(5 \times 5 \times 5 \mathrm{~cm}^{3}\right)$. The CUORE sensitivity to $0 v$ DBD is currently limited by the background produced by $\alpha$ decays originating on the surface of the inert materials facing the detector. It was observed [3] that $\beta$ particles, differently from $\alpha$ s, have sufficient energy to emit Cherenkov light in $\mathrm{TeO}_{2}$. The signal is however of only about $100 \mathrm{eV}$ [4] and therefore Light Detectors (LDs) with energy resolution below $20 \mathrm{eV}$ are needed to achieve a S/N ratio of 4-5 and a satisfactory $\alpha$ background rejection.

LUCIFER will have a much smaller background compared to CUORE (zero background limit in the region of interest). This result will be obtained using scintillating crystals as bolometers and isotpes with high Q-values (e.g. ZnSe crystals enriched in ${ }^{82} \mathrm{Se}$ or $\mathrm{ZnMoO}_{4}$ crystal enriched in ${ }^{100} \mathrm{Mo}$ ). The LDs needed to obtain a good particle discrimination for this experiment has to be less performing (energy resolution of about $100 \mathrm{eV}$ ). They were already realized by the collaboration and consist of $5 \mathrm{~cm}$ diameter germanium slabs operated as bolometers [6]. On the other hand LUCIFER would receive an important benefit from a LD with better resolution. In fact, with a energy resolution of $20 \mathrm{eV}$ RMS it would have enough sensitivity to discriminate nuclear recoils from $\beta / \gamma$ background in the region of spectrum where the signal from WIMP Dark Matter interaction is expected $(0 / 20 \mathrm{keV})[5]$.

\section{A new way for light detectors: CALDER}

CALDER (Cryogenic wide-Area Light Detectors with Excellent Resolution) is a R\&D started in 2014. The aim of the project is to develop large area, high energy resolution cryogenic LD, that are extremely needed to enhance rare events experiments as shown in previous section. The current state of the art in this field is the CRESST LD [7]. This detector, realised with Transition Edge Sensors, shows an excellent resolution (lower than $30 \mathrm{eV}$ RMS), but suffers from reliability and reproducibility problems. Moreover the multiplexing readout system for a TES array is expensive and quite complex to realize.

We chose to develop our LD, using a novel technologies, called Kinetic Inductance Detectors (KIDs) [8]. KIDs are superconducting microresonator devices with very high Q factors $\left(10^{4}-\right.$ 

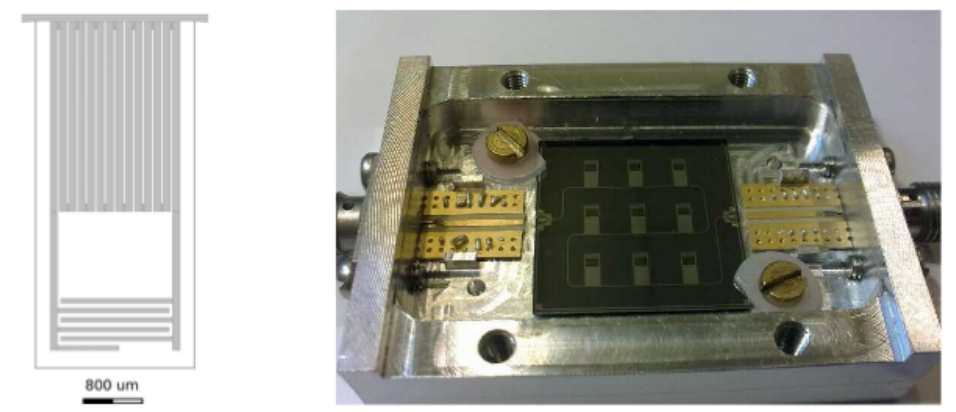

Figure 1: Left. A sketch of our resonator design. Right. The first 9-pixel prototype in his holder.

$10^{6}$ ). The main advantages of this technology are represented by the excellent reliability, simple signal readout and easy scalability thanks to the intrinsic frequency multiplexing. The main issue regarding the adoption of KIDs is represented by the need to cover a large surface $\left(\approx 25 \mathrm{~cm}^{2}\right)$ with few pixels (4-10), because the largest possible size of a KID without an important loss of sensitivity and bandwidth is about $5 \mathrm{~mm} \times 5 \mathrm{~mm}$.

We are implementing an indirect phonon-mediated detection [9]: photons are absorbed in the substrate of the KID, generating a population of athermal phonons able to break Cooper pairs in the resonator. Using this approach, it is possible to reach high efficiency (up to $50 \%$ ) with relatively small filling ratio (about $10 \%$ ) if the main loss mechanisms (thermalization and escape of the phonons through the holder) are properly minimized. Our starting point is the the work of Moore [10], who developed a similar detector to be used for Dark Matter searches. With respect to this work we are not interested in resolving the position of interaction. This will allow us to enhance the merit factor of the detectors, realizing slower devices with higher sensitivity.

\section{Status of the Project}

We have recently completed the test of the first prototype of LD, produced at IFN of CNR (Rome). A $300 \mu \mathrm{m}$ thick, $2 \times 2 \mathrm{~cm}^{2}$ wide silicon substrate is sampled by 9 KID sensors, made with $40 \mathrm{~nm}$ thick aluminum deposited by electron gun. The lithography is performed using an EBL with a lift-off approach. The single pixel (left part of fig 1) has an active area of $2.3 \mathrm{~mm}^{2}$ (total filling ratio of $5 \%$ ) and resonates at $2.5 \mathrm{GHz}$ with a quality factor of $3 \times 10^{4}$. This first array design was realized after studies to optimize detector parameter as the volume of the absorber substrate, the thickness of the resonator and the filling ratio. The detector's holder (see right panel of fig. 1) was designed to minimize the loss of energy due to the escape of the phonons through the supports.

During the first measures we were not able to test completely the device, since our electronics readout and analysis tools were still not well developed. We were able instead to study the shape of the resonances (see the left part of fig. 2). Using the readout system [11] developed for NIKA [12], we were also able to trigger CRs and light signals, sent through an optical fiber (respectively the red and black line in the right panel of fig. 2). The sensitivity of the device was strongly deteriorated by the broken feedline and we expect to improve significantly our sensitivity with the 2nd generation 

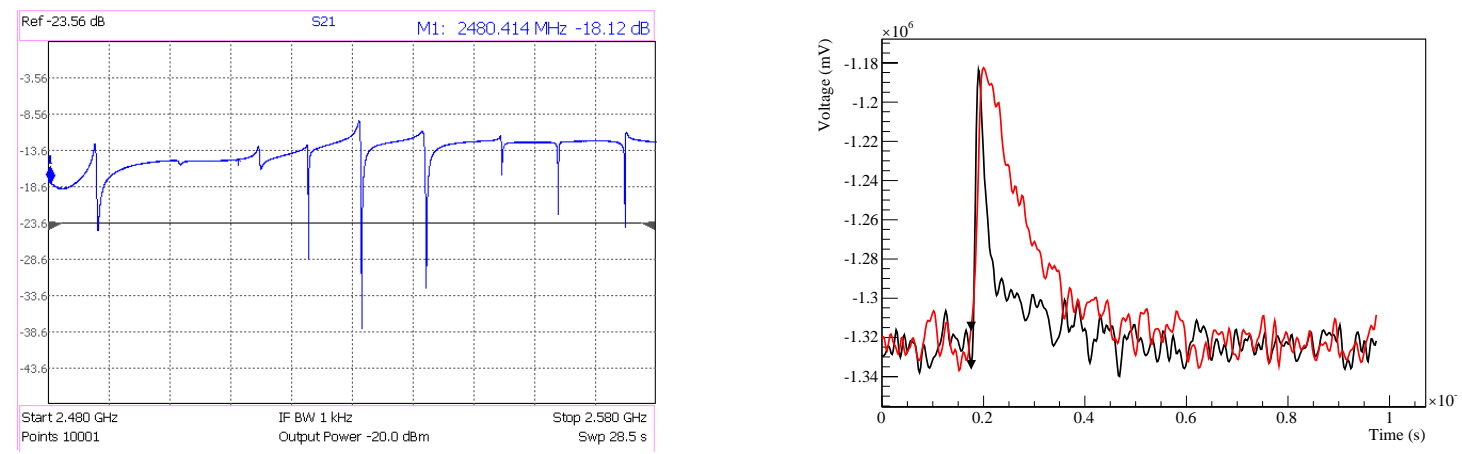

Figure 2: Left. The 9 resonances measured in our first prototype Right. Two pulses caused by a Cosmic ray (red) and by a light signal (black).

of devices, that is currently in production. The new sample will include a simplification of the geometry of the array (from 9 to 4 pixels) in order to minimize the effect of the crosstalk, that is well evident in fig. 2 . We will also try to increase the merit factor of the resonator up to $10^{5}$. The 2nd generation of devices will be tested in the next weeks, profiting of the fully operational readout electronics and of the model of response of the resonator to pulses, developed during the first tests. At the same time we are now beginning the study of materials different from aluminum (e.g. non stoichiometric TiN or multilayer Ti TiN), since their lower critical temperature and higher kinetic inductance will contribute to enhance the energy resolution of our LDs.

\section{Acknowledgments}

The CALDER experiment is funded by the European Research Council under the European Unions Seventh Framework Programme (FP7) / ERC grant agreement n. 335359.

\section{References}

[1] C. Arnaboldi et al., Nucl. Instrum. Methods A518, 775-798 (2004)

[2] J.W. Beeman et al., Advances in High Energy Physics 2013 (2013)

[3] T. Tabarelli de Fatis, Eur. Phys. J. C65 (2010) 359.

[4] N. Casali, M. Vignati et al. arxiv:1403.5528

[5] J.W. Beeman et al., JINST 8, P05021 (2013)

[6] J.W. Beeman et al., JINST 8, P07021 (2013)

[7] G. Angloher et al., Eur. Phys. J. C72, 1971 (2012)

[8] P. Day et al., Nature 425, 817-821 (2003)

[9] L. Swenson et al., Appl. Phys. Lett. 96, 263511 (2010)

[10] D.C. Moore et al., Appl. Phys. Lett. 100, 262601 (2012)

[11] O. Bourrion et al., JINST 6, P06012 (2011)

[12] A. Monfardini et al., ApJS 194, 24 (2011) 